\title{
THE HEALTH LITERACY ENVIRONMENT OF A REGIONAL AUSTRALIAN ELECTIVE SURGERY ACCESS UNIT: CONSUMER PERSPECTIVES FROM PRE-ADMISSION TO POST-DISCHARGE
}

\author{
Sarah Neil', Kylie Murphy2, Glenda Chapman' \\ 1. Albury Wodonga Health, Wodonga, Victoria, Australia \\ 2. Charles Sturt University - School of Community Health, Albury, New South Wale, Australia
}

Correspondence: smneil2001@gmail.com

\begin{abstract}
\section{BACKGROUND}

Healthcare services should conscientiously ensure their health literacy environment (HLE) supports wayfinding and provides comprehensible health information. Despite the increasing focus on the importance of evaluating and enhancing the HLE, consumer perspectives about HLE barriers and enablers have received limited attention in the published literature.
\end{abstract}

\section{OBJECTIVE}

This study aimed to identify barriers and enablers in the HLE of the Elective Surgery Access Unit (ESAU) at Albury Wodonga Health in regional south-east Australia.

\section{METHODS}

Three consumers participated in the study. Two of these participants completed a wayfinding interview, verbalizing the barriers and enablers encountered during wayfinding from the nearest carpark to the ESAU. All participants reviewed samples of written materials for ESAU consumers. Two participants, who had been discharged, commented on whether any important information was overlooked, from a post-discharge perspective. The data was categorized into inter-related themes within broader overarching domains.

\section{RESULTS}

The helpfulness of the physical environment was one domain, involving three themes: signage, parking, and visual cues. The helpfulness of written information was another domain, involving three themes: comprehensiveness, readability and relevance. A third overlapping domain was: the importance of verbal information-giving. This domain also involved three themes: the importance of a phone number to seek assistance, a clearly identifiable reception area, and in-person communication.

\section{CONCLUSIONS}

The insights of these three service users can inform health services trying to enhance access for everyone needing healthcare. If more Australian health services reviewed their HLE, the findings could inform organizational improvements towards safer, more efficient, and higher quality healthcare.

\section{KEYWORDS}

Health Literacy, Environment, Wayfinding, Health Information, Evaluation

\section{INTRODUCTION}

Health literacy is an umbrella term which includes individual health literacy and the health literacy environment. [1] Individual health literacy involves all the skills and attributes of a person which enable them to manage their own health. [1] As our understanding of health literacy issues has evolved, it has become apparent that the nature of the healthcare system supports or impedes consumers in managing their health. [1] Acknowledging this relationship 
between the person and their healthcare environment, the health literacy environment (HLE) is now considered an important component of health literacy. [1] The HLE includes health information, healthcare workers, the physical environment and the political context of healthcare. [1] Recognition that health literacy demands contribute to barriers, inequalities and fragmentation of healthcare has led to an increased focus on the HLE. This study focused on two aspects of the HLE: written information and wayfinding.

Few existing studies have evaluated wayfinding and written information in healthcare settings. Groene and Rudd [2] evaluated wayfinding and written information at 10 hospitals in Spain, identifying barriers including inconsistent or incorrect signage. An American study by Pati and colleagues [3] identified elements of the physical environment which influenced wayfinding, including maps, signs and clustering of destinations.

In Australia, Johnson [4] used the Health Literacy Environment Activity Packet [5] to evaluate the HLE of one Australian health service. Two consumers and one hospital employee found that wayfinding barriers contributed to the need for verbal directions. Two other Australian studies explored the health literacy of written consumer information. Byles, Chiarelli, and Hacker [6] recommended ensuring clarity, brevity and accuracy of written information. Similarly, a study by Dickinson and colleagues [7] highlighted the importance of verbal information from service providers in addition to written materials.

Much of the existing research lacks consumer voice, particularly consumers with low health literacy. The scarcity of HLE research in general limits the confidence with which existing findings can be transferred to other population groups and healthcare settings.

This study aimed to identify barriers and enablers in the HLE of an Elective Surgery Access Unit (ESAU) based at one hospital in regional south-east Australia. The ESAU receives referrals, books elective surgical procedures, and provides pre-clinical information to consumers. The information gathered was intended for use by ESAU managers and clinicians to improve universal access to the service by improving the HLE. The findings were also intended to prompt possible HLE improvements in other similar services.

\section{DESIGN}

Responding to the above-noted lack of consumer input in existing HLE research, and in line with national safety and quality health service standards [8], feedback was sought from three ESAU consumers. A wayfinding interview identified navigational barriers and enablers. A writteninformation interview involved the participant reviewing documents provided by the ESAU and included postdischarge questions which explored the participant's overall view of information they were provided about their procedure.

Ethics approval for this study was obtained from two local Human Research Ethics Committees (HREC).

\section{SAMPLING}

Participants were invited from one surgical group in the region at the time of their referral to the ESAU. Participants were invited to take part in this study only if they: were unfamiliar with the ESAU and unable to find their way to the service by memory; had adequate written and verbal English skills to understand the requirements of the study and give their informed consent; could mobilize independently, without the help of a carer; and were aged 18 years or over. Three participants responded and this was believed to be adequate to uncover some key barriers and enablers experienced by ESAU clients, without overburdening ESAU clients.

\section{PARTICIPANTS}

Three participants were recruited. Two participants were male, and one was female. All participants were aged over 65 years. However, only $\mathrm{Pl}$ accepted the invitation to participate through the recruitment method described above. Pl was also unable to share their post-discharge perspective as they remained on the waitlist for surgery when the data collection period concluded. Therefore, other participants were recruited via alternative means. P2 was referred to the study by the specialist group but they had undergone their elective surgery six months prior. P2 completed the written information interview, including the post-discharge questions, but not the wayfinding interview as they were already familiar with the ESAU's location. P3 was known to the primary researcher and self-referred to the study four months after having surgery at the service. P3 
participated in both interviews, including the postdischarge questions. They had not previously attended the

ESAU as their elective procedure was booked during an inpatient stay.

\section{DATA COLLECTION}

Two interviews were held with each participant, unless described otherwise above. All interviews were voicerecorded and transcribed. In the wayfinding interview, the participant verbally described their experience while navigating their way to ESAU alongside the researcher. This uncovered real-time data about what assisted them to find their way and what caused difficulty. This is consistent with the strategy used in the study by Johnson. [4]

In the written information interview, participants reviewed and commented on the clarity and readability of four documents commonly provided to ESAU consumers. This interview aimed to identify barriers and enablers in the written information from a consumer perspective. The postdischarge questions included: "Should other information have been included in these documents which was not?"
The purpose of these questions was to consider whether the information provided was comprehensive, in hindsight.

\section{DATA ANALYSIS}

Concepts in the participants' feedback were initially grouped into clusters of related points and labelled as themes, in a process of open coding. $[9,10]$ These themes were then grouped together based on their relevance to emergent, overarching domains; a process called axial coding. [10] Constant comparison was used in that new data was compared with existing themes and new themes were created as needed. [9] The ultimate intention was to parsimoniously subsume the final set of themes into the fewest, simplest and clearest domains possible, providing a useful framework for understanding consumer experiences of barriers and enablers when accessing the ESAU.

\section{RESULTS}

The data was analysed and collated into nine inter-related themes, which were subsequently categorized into three overall domains which were not mutually exclusive. These domains and themes are depicted in Figure 1.

FIGURE 1. DOMAINS AND THEMES ARISING FROM THE CONSUMER INTERVIEWS

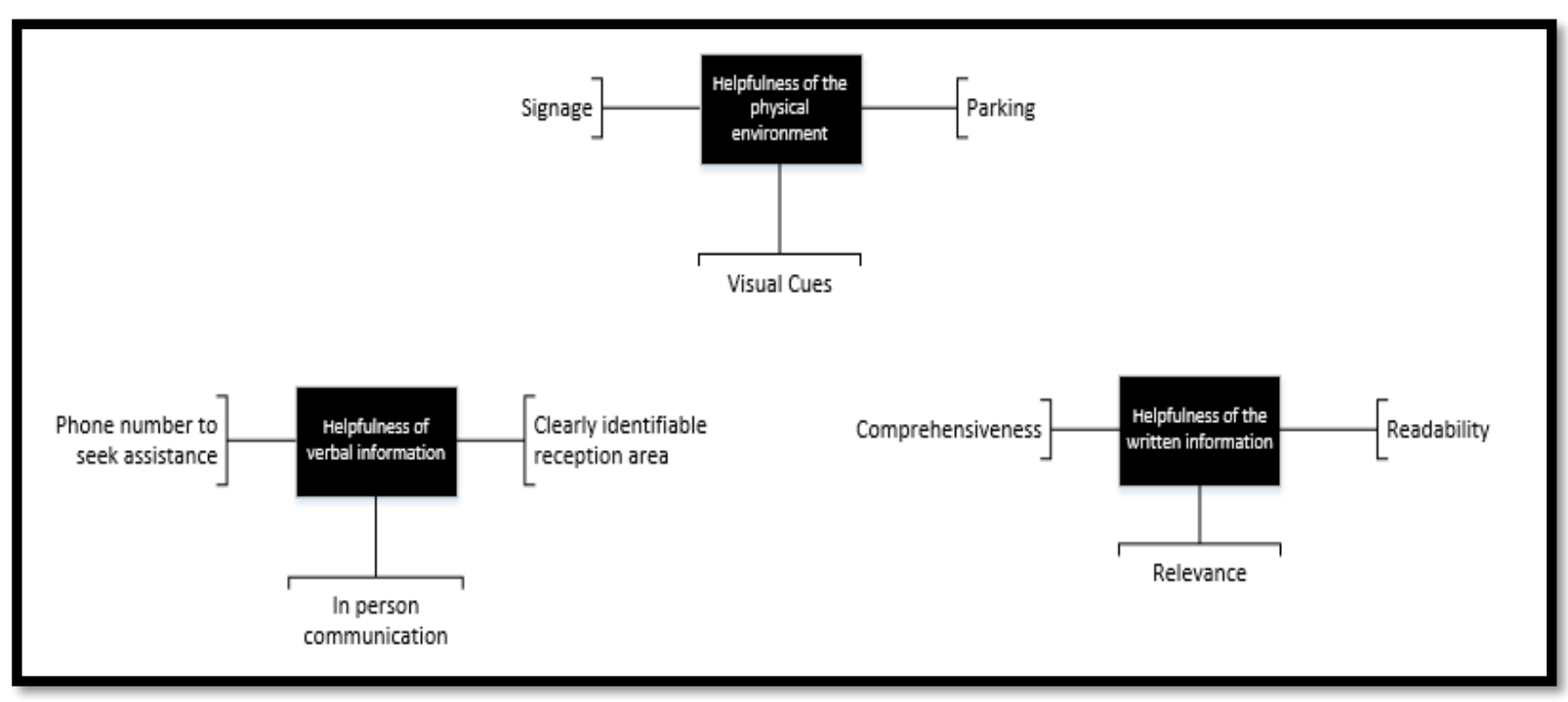




\section{HELPFULNESS OF THE PHYSICAL ENVIRONMENT}

Parking. When asked about wayfinding to the main entrance, participants invariably commented on the limited parking and the difficulty this created in terms of accessing the ESAU.

"Parking's really bad most days." PI

Signage. Two consumers commented that the signage supported their wayfinding.

"It's good signage." PI

"you see the two signs for pre-admission or for the wards if you're visiting, excellent." P3

As a possible improvement, it was suggested that signs and directional prompts, such as arrows and doorways, to a particular destination should be colour coded.

"...you just keep following the red arrow, red sign, red arrows... if it's red, it stays red. Stay on the colour code it indicates." P3

The term 'Pre-admission' on signage was identified as unhelpful.

"It doesn't say 'referrals' to the lay person." P3

Visual cues. Visual cues are indicators in the built environment which influence navigation. The pebble-crete pathway from the carpark to main reception assisted wayfinding because it differed from nearby road surfaces.

"it's obvious this is the path you walk on. It's not confusable with other pathways." P3

Two participants commented that the main entrance, which features a large awning visible from the car park, was easy to find. The entrance has automatic glass doors, through which the reception area is visible.

"you don't need a sign that big because it's set up as a reception point and we're so used to what a reception point is to look at, you don't have to have a label on a dog to know it's a dog." P3

However, Pl commented that the main reception and Emergency Department entrances were close together, which created confusion about which to enter, but signage assisted with overcoming this confusion.

\section{HELPFULNESS OF VERBAL INFORMATION}

In-person communication. Verbal information received from service providers in addition to written information was highlighted as valuable.

"...they [ESAU staff] explained what was going on well." Pl

Clearly identifiable reception area. Participants invariably commented on the importance of the reception desk for seeking verbal directions.

"There's no problem just asking at the front desk, which is right inside the front doors, you'd have to be blind Freddy to miss it." P3

Phone number to seek clarification. Two participants highlighted the importance of having a phone number in the written information to seek clarification if needed.

"If you come across something that you weren't sure of, there's provisions there for you to double check it if you needed to." P2

\section{HELPFULNESS OF WRITTEN INFORMATION}

Readability. P3 felt that all the written documents contained information which was unclear.

"...none of that would be easy for a stumbling reader, they'd have to get someone to help with all of that..." P3

Two participants reported that the directions to confirm the booking were unclear.

"So you have to ring to confirm your date then? Is that what it's saying here?" $P I$

P3 recommended that directions should clearly state what action is required first, followed by instructions, and then any reasons or consequences.

Two participants reported that jargon was unhelpful; for example, 'post-operatively'.

"Not much good 'em putting words in there that you can't spell... or that you can't pronounce." P2

P3 felt the phrase 'ready for care' was ambiguous and created anxiety about who decides 'readiness' and whether being 'unready' meant going to the bottom of the waiting list. 
"Is the specialist making the decision 'you're not ready for care? ... We're ready to go and you're not, well you go back down the list'." P3

P3 recommended reducing and simplifying images or characters on the page to reduce visual distraction.

"Anything...that robs you of your absolute attention to detail that's required is a bad thing." P3

Two participants identified that presenting lists of recommended and restricted foods in columns made the information easy to read and use.

"You can sort of run a pen through that and say well I'm not even looking at that, this is the only stuff I can eat." P2

Comprehensiveness. Several pieces of information in the written materials were felt by the participants to be key. First, it was important to know your level of priority and what that designation meant.

"lets you know if you're not, not bad you'll be put on the waiting list... if you're urgent you'll go straight in." P2

Second, practical information such as dates, place names and contact details were deemed important.

"The contact details are the (useful) ones I think." P2

Third, directions for actions the reader was required to complete were considered important.

...that tells me what I can eat." PI

No key information was viewed to be lacking by any participant.

Relevance. Two participants felt that excessive detail in the written information was unhelpful or confusing.

"It might sound more polite, the way it was written, but this is not a literature essay, this is a set of instructions for you to get knowledge from straight away." P3

P3 also noted that some explanations were rambling.

"... "as you may be aware...each patient will be assigned an urgency category'... who cares if you're aware. You may not be but if you say 'I wasn't' ... it doesn't matter." P3
However, P2 did not feel that unnecessary information clouded the key points.

"...the basics are there without any humble jumble." P2

\section{DISCUSSION}

The aim of this study was to identify health literacy barriers and enablers from an Elective Surgery Access Unit (ESAU) consumer perspective. Interviews uncovered elements of the physical environment which impacted on navigation and important considerations for enhancing the helpfulness of written information. An emergent, interwoven theme was the importance of verbal information-giving.

\section{WAYFINDING}

The importance of adequate parking to support wayfinding was clear as it was invariably the first thing participants mentioned. The use of different path surfaces, such as concrete or pebble-crete, clearly distinguished the path to the hospital's main reception. This finding supports current guidelines about delineating pathways to support wayfinding. [1 1]

Current guidelines recommend that entrances should have unique features to assist navigation to the desired point of entry. [12] The findings of this study, however, raise the question of whether typical visual cues around entry points might be culturally sensitive. P1 identified as culturally and linguistically diverse (CALD) and reported difficulty distinguishing the main entrance from the emergency department entrance nearby. In contrast, P3 was born in Australia and reported no difficulty identifying the main entrance. $33.3 \%$ of Australian residents were born overseas [13], therefore care must be taken to avoid making assumptions about cultural knowledge.

Hospital departments rarely differ from one another in appearance, so signage and landmarks are necessary to support navigation. [11] Participants reported that signage generally enabled wayfinding; however, inconsistent place names were a barrier. Participants were sent to the "ESAU" but signage to that service read "Pre-Admission". A wayfinding review of ten hospitals in Spain also found that inconsistent terminology on signage impeded wayfinding. [2] It is important to ensure consistency of place names across referrals, signage and verbal directions. [12] One participant in the current study recommended colour- 
coding signage. For example, providing all signage to the ESAU in purple, a purple line on the floor and purple arrows leading to the purple doors of the ESAU. The consumer simply follows the purple cues. The idea has practical merit; indeed this type of wayfinding system is used in other public spaces, including Melbourne's 282 acre Fawkner Memorial Park. [14]

Participants in this study mentioned the importance of seeking verbal directions at reception. In a similar study by Johnson, participants sought directions but reported an unwillingness to bother staff. [4] In the present study, participants unreservedly sought directions at reception, suggesting that a clearly identifiable reception is a socially acceptable point for obtaining verbal directions.

\section{VERBAL INFORMATION-GIVING}

The Australian Charter of Healthcare Rights states consumers have a right to be informed about their care. [15] When asked in the post-discharge questions whether they felt adequately informed about their procedure, participants spoke about verbal information received from service providers. Participants also made reference to the value of having a phone number to seek verbal clarification, which is consistent with recommendations that consumers should have multiple opportunities to receive information. [1] Verbal information, in addition to written materials, was considered essential for feeling informed, which is consistent with the findings of Dickinson and colleagues. [7]

\section{WRITTEN INFORMATION-GIVING}

Participants in the present study emphasised the importance of writing in plain English. Other research similarly points to difficulties associated with comprehending medical or scientific terminology, even for highly literate readers. [16] In the present study, ambiguous terminology was an identified barrier, creating confusion and anxiety. Participants in a larger Australian study also recommended rephrasing ambiguous statements. [6] Unclear instructions noted by participants in the present study are concerning because misunderstood instructions can lead to inadvertent non-adherence to recommendations. [17] One participant recommended three-step instructions: a clear direction to the reader that the action must be completed; instructions for completing the action; and any relevant reasons or consequences. Participants also reported that advice in simple, tabulated form enabled them to better understand and act on recommendations. In general, participants reported that excessive detail and repetition were barriers. Other studies have recommended concise wording [6, 7], with consumers describing too much text as demotivating, intimidating or even frightening. [16]

One participant reported that images unrelated to text were highly distracting. A past study which compared the views of low and high literacy readers found that participants with low literacy tended to be receptive to images on the page, while high literacy participants were generally critical. [16] The present study did not assess literacy levels, so it is uncertain why images were a particular distraction for one participant. In view of similar recommendations to reduce crowded 'busy' formatting [6], it seems reasonable to assume that unless the images reinforce or replace text, minimising graphic distraction in written information is wise.

\section{LIMITATIONS}

Four limitations apply to this study. First, two participants were recruited post-discharge potentially skewing the findings towards a post-discharge perspective. Secondly, the small sample size precludes confident generalization of the findings to other ESAU consumers. Thirdly, self-selection in this study probably resulted in an over-representation of people with higher health literacy and/or general selfconfidence levels. Finally, data saturation was not reached in this study, which compromises the comprehensiveness of the findings.

\section{CONCLUSION}

This study uncovered barriers to wayfinding including limited parking, jargon on signage and unclear visual cues. Identified wayfinding enablers included distinctive pathways, an identifiable reception point, and access to verbal directions. Identified enablers for written consumer information included the use of plain English, succinct expression, and lists or tables. Barriers identified included distracting images, jargon, excessive text, and unclear instructions. Verbal information was found to support both wayfinding and comprehension of written materials.

It is not unreasonable to assume that the themes identified in this study are applicable to other healthcare services; particularly those supported by the findings of similar 
studies. The findings of this study are significant because they suggest that other services should conduct their own consumer-centered HLE evaluations. Publication of the findings of such evaluations would increase awareness of the full range of factors that impact on the accessibility and quality of care consumers are receiving, which may in turn lead to important improvements.

Future HLE evaluations should focus on specific healthcare departments and investigate both wayfinding and information provision, like in this study; but aim to recruit larger consumer numbers, with as many interviews as are required to reach data saturation. Future research could also explore a range of issues in relation to maximizing HLEs. Is colour-coding signs and directional prompts a viable wayfinding strategy for healthcare services? What are consumer preferences around seeking verbal information, and are these influenced by demographic variables? Continued HLE research could lead to cost-effective improvements in the quality and effectiveness of Australian healthcare services.

\section{References}

1. Australian Commission on Safety and Quality in Health Care. Health literacy: Taking action to improve safety and quality. Sydney: ACSQHC; 2014.

2. Groene RO, Rudd RE. Results of a feasability study to assess the health literacy environment: Navigation, written, and oral communication in 10 hospitals in Catalonia, Spain. J Commun Healthc. 2011;4(4):22737.

3. Pati D, Harvey TE, Willis DA. Identifying elements of the health care environment that contribute to wayfinding. HERD. 2015;8(3):44-67.

4. Johnson A. First impressions: Towards becoming a health-literate health service. Aust Health Rev. 2014;38(2):190-3.

5. Rudd RE. The health literacy environment activity packet: First impressions and a walking interview. Online tools: Health Literacy Studies; 2010.

6. Byles JE, Chiarelli $\mathrm{P}$, Hacker $\mathrm{AH}$. The evaluation of print material used within three models of continence care. Australian and New Zealand Continence Journal. 2006;12(3):75

7. Dickinson R, Hamrosi K, Knapp P, Aslani P, Sowter J, Krass I, et al. Suits you? a qualitative study exploring preferences regarding the tailoring of consumer medicines information. Int J Pharm Pract. 2013; 21:20715.

8. Australian Commission on Safety and Quality in Health Care. Safety and quality improvement guide standard 2: Partnering with consumers. Sydney: ACSQHC; 2012.

9. Bryant A. Grounded Theory and Grounded Theorizing: Pragmatism in Research Practice. New York: Oxford University Press; 2017.

10. Given L. The SAGE Encyclopedia of Qualitative Research Methods. London: SAGE Publications, Inc; 2008.

11. Allison D. Hospital as city: employing urban design strategies for effective wayfinding. Health Facilities Management. 2007;20(6):61-5.

12. NSW Ministry of Health. Wayfinding for Healthcare Facilities. http://www 1.health.nsw.gov.au: NSW Government; 2014.

13. Statistics ABo. 2016 Census quickstats : People cultural \& language diversity. 2017.

14. Greater Metropolitan Cemeteries Trust. Fawkner Memorial Park: Park map Melbourne: Greater Metropolitan Cemeteries Trust; 2015 [Available from: http://gmct.com.au/our-locations/fawkner-memorialpark/.

15. Australian Commission on Safety and Quality in Health Care. Australian charter of healthcare rights. In: Australian commission on safety and quality in health care, editor. Sydney: ACSQHC; 2008.

16. Smith SK, Trevena L, Nutbeam D, Barratt A, McCaffery KJ. Information needs and preferences of low and high literacy consumers for decisions about colorectal cancer screening: Utilizing a linguistic model. Health Expectations. 2008; 11:123-36.

17. Koh HK, Brach C, Harris LM, Parchman ML. A proposed 'health literate care model' would constitute a systems approach to improving patients' engagement in care. Health Affairs. 2013;32(2):357-67. 